\title{
Artemisinin reduces cell proliferation and induces apoptosis in neuroblastoma
}

\author{
SHUNQIN ZHU ${ }^{1-3^{*}}$, WANHONG LIU ${ }^{1,4^{*}}$, XIAOXUE KE ${ }^{2}, \mathrm{JIFU} \mathrm{LI}^{2}$, \\ RENJIAN HU ${ }^{2}$, HONGJUAN CUI ${ }^{2}$ and GUANBIN SONG ${ }^{1}$
}

\begin{abstract}
${ }^{1}$ Key Laboratory of Biorheological Science and Technology, Ministry of Education, College of Bioengineering, Chongqing University, Chongqing; ${ }^{2}$ State Key Laboratory of Silkworm Genome Biology, Southwest University, Chongqing; ${ }^{3}$ College of Life Science, Southwest University, Chongqing; ${ }^{4}$ School of Chemisty and Chemical Engineering, Chongqing University of Science and Technology, Chongqing, P.R. China
\end{abstract}

Received April 3, 2014; Accepted May 15, 2014

DOI: $10.3892 /$ or.2014.3323

\begin{abstract}
Artemisinin, a natural product from the Chinese medicinal plant, Artemisia annua L., is commonly used in the treatment of malaria, and has recently been reported to have potent anticancer activity in various types of human tumors. Yet, the effect of artemisinin on neuroblastoma is still unclear. In the present study, we aimed to investigate the effects of artemisinin on neuroblastoma cells. We observed that artemisinin significantly inhibited cell growth and proliferation, and caused cell cycle arrest in the G1 phase in neuroblastoma cell lines. Annexin V-FITC/PI staining assay revealed that artemisinin markedly induced apoptosis. Soft agar assays revealed that artemisinin suppressed the ability of clonogenic formation of neuroblastoma cells and a xenograft study in NOD/SCID mice showed that artemisinin inhibited tumor growth and development in vivo. Therefore, our results suggest that the Chinese medicine artemisinin could serve as a novel potential therapeutic agent in the treatment of neuroblastoma.
\end{abstract}

Correspondence to: Professor Hongjuan Cui, State Key Laboratory of Silkworm Genome Biology, Southwest University, 2 Tiansheng Road, Beibei, Chongqing 400716, P.R. China

E-mail: hongjuan.cui@gmail.com; hcui@swu.edu.cn

Professor Guanbin Song, Key Laboratory of Biorheological Science and Technology, Ministry of Education, College of Bioengineering, Chongqing University, 174 Shapingba Street, Shapingba, Chongqing 400044, P.R. China

E-mail: song@cqu.edu.cn

${ }^{*}$ Contributed equally

Key words: artemisinin, neuroblastoma, cell growth, proliferation, apoptosis, cell cycle

\section{Introduction}

Neuroblastoma is a common childhood malignant tumor of neural crest origin, arising in the paravertebral sympathetic ganglia and the adrenal medulla (1). The clinical characteristics of neuroblastoma include extreme heterogeneity, easy transfer and high malignant potential $(2,3)$. Neuroblastoma accounts for approximately $10 \%$ of all pediatric cancers and $15 \%$ of cancer-related deaths in children (3-9). Although conventional therapies that are based on surgery, chemotherapy and radiotherapy are available, these approaches have limited efficacy in many cases (8). Therapy failure is generally caused by acquired chemoresistance or high toxicity in neuroblastoma patients. The major challenge in neuroblastoma treatment is the identification and development of new drugs with specific effects (10).

Natural plant compounds represent a large group of untapped potential medicines. Various chemical compounds extracted from natural plants have been reported to have high efficacy and few side-effects (11). Artemisinin (ART), also known as Qinghaosu, was isolated from the Chinese herb Artemisia annua L. (Qinghao), a type of wormwood native to Asia. It was discovered in the early 1970s by Tu Youyou $(12,13)$. It has been approved as a potent anti-malarial agent by the Food and Drug Administration (FDA), and artemisinin is commonly used in the clinical management of malaria $(14,15)$. Recently, it was reported that artemisinin or its derivatives have anticancer activity in many types of cancers, such as in multiple tumors $(16,17)$, breast cancer $(18)$, hepatocellular carcinoma (19), leukemia (20), prostate cancer (16) and oral cancer (21). Yet, the role of artemisinin in neuroblastoma has not been well characterized.

In the present study, we investigated the potential role of artemisinin in neuroblastoma cells. We determined that artemisinin induces the inhibition of cell proliferation and induces the apoptosis of the neuroblastoma cells in vitro. Moreover, we also demonstrated that artemisinin suppressed clonogenic formation ability and xenograft tumor growth in NOD/SCID mouse models. As a natural product, artemisinin has been confirmed to have no or fewer side effects in the treatment of 
malaria. Thus, artemisinin could be used as a novel potential drug for the treatment of neuroblastoma.

\section{Materials and methods}

Cell culture. Human neuroblastoma cell lines SK-N-AS, SK-N-DZ and SHEP1 were grown in Dulbecco's modified Eagle's medium (DMEM) supplemented with $10 \%$ fetal bovine serum (FBS) and 1\% antibiotics penicillin and streptomycin $(\mathrm{P} / \mathrm{S})$. $\mathrm{BE}(2)-\mathrm{C}$ cells were cultured in a 1:1 mixture of DMEM and Ham's nutrient mixture F12 (DMEM/F12), supplemented with $10 \%$ FBS and $1 \%$ antibiotics (P/S). All four cell lines were purchased from ATCC (Manassas, VA, USA). The growth media, antibiotics and FBS were purchased from Life Technologies. All cells were cultured at $37^{\circ} \mathrm{C}$ in a $5 \% \mathrm{CO}_{2}$ humidified incubator.

Cell growth and proliferation assay. Artemisinin was dissolved in DMSO. The cell growth curve was detected using the Cell Counting Kit- 8 assay (CCK-8; Beyotime, China). Briefly, approximately 1,000 cells in $200 \mu \mathrm{l}$ medium were seeded in 96-well plates and incubated with artemisinin at concentrations of $100,200,300$ and $400 \mu \mathrm{M}$; DMSO was used as a control. CCK-8 (20 $\mu \mathrm{l})$ was added and incubated for $2 \mathrm{~h}$ every 2 days, and the absorbance at $450 \mathrm{~nm}$ was used to detect metabolically intact cells according to the manufacturer's protocol. Cells were exposed to $300 \mu \mathrm{M}$ artemisinin or DMSO for $72 \mathrm{~h}$, and cell morphologic examination was carried out using an inverted microscope (TS100, Nikon). Then adherent cells were collected and washed with ice-cold PBS. The sample obtained was analyzed by the TC10 ${ }^{\mathrm{TM}}$ Automated Cell Counter (BioRad, Hercules, CA, USA).

Immunofluorescence staining. Cells were grown on coverslips and treated with either DMSO or $300 \mu \mathrm{M}$ artemisinin for $72 \mathrm{~h}$. Cells were washed with PBS, fixed for $20 \mathrm{~min}$ in $4 \%$ paraformaldehyde (PFA), and permeabilized with $0.3 \%$ Triton X-100 for 5 min. The cells were blocked with $10 \%$ goat serum in PBS for $1 \mathrm{~h}$, incubated with a primary rat antibody against Ki67 (1:300, 556003; BD) in blocking buffer for $1 \mathrm{~h}$ at room temperature, and then incubated with the secondary antibody Alexa Fluor 595 goat anti-mouse IgG (H+L) (1:2,000; A-21422; Invitrogen). DAPI (300 nM) in PBS was used for nuclear staining. Cells were examined using a Nikon microscope (80i) with Image-Pro Plus software for image analysis. Ki67 uptake was calculated in 10 microscopic fields.

Cell cycle assay. Cells were plated in $10-\mathrm{cm}$ plates and treated either with $300 \mu \mathrm{M}$ artemisinin or DMSO. After $72 \mathrm{~h}$ of treatment, cells were fixed with $70 \%$ cold ethanol, stained with propidium iodide (PI) and analyzed by flow cytometry (Accuri C6; BD). The data were analyzed with ModfitLT software.

Western blot analysis. Cells treated with artemisinin for $72 \mathrm{~h}$ were harvested and suspended in RIPA lysis buffer (Beyotime, China). Protein concentrations were determined with the Enhanced BCA protein assay kit (Beyotime). Sixty micrograms of protein was separated on $10 \%$ sodium dodecyl sulfate polyacrylamide gels (SDS-PAGE), transferred to PVDF membranes, and analysis was performed with the primary antibodies including anti-cyclinD1 (1:500; Santa Cruz), anti- $\alpha$-tubulin (1:2,000; Sigma-Aldrich), anti-CDK4 (1:500; Santa Cruz), anti-cyclinE2 (1:500; Santa Cruz), anti-cyclinB1 (1:500; Santa Cruz). Horseradish peroxidase-conjugated goat anti-mouse $(1: 20,000)$ and rabbit anti-goat $(1: 10,000)$ immunoglobulin G (IgG; KPL, Gaithersburg, MD, USA) were used as secondary antibodies. Proteins were visualized with BeyoECL Plus (Beyotime, China).

Cell death and apoptosis assay. For cell death assay, all cells grown to $60-70 \%$ confluency were treated with $300 \mu \mathrm{M}$ artemisinin for $72 \mathrm{~h}$, and cells were treated with DMSO as control. After treatment, adherent and floating cells were collected by centrifugation, and then the sample obtained was analyzed using the $\mathrm{TC} 10^{\mathrm{TM}}$ Automated Cell Counter. Cell counting was carried out using trypan blue dye (145-0021, Bio-Rad) staining as previously described (22). The apoptotic ratios of the cells were determined with the Annexin V-FITC apoptosis detection kit (Sigma). Briefly, after a 72-h treatment with DMSO or $300 \mu \mathrm{M}$ artemisinin, the cells were collected and washed twice with cold PBS buffer, resuspended in $100 \mu \mathrm{l}$ of binding buffer, and incubated with $5 \mu 1$ of Annexin $\mathrm{V}$ conjugated to FITC and $10 \mu \mathrm{l}$ PI for $15 \mathrm{~min}$ at room temperature. Cell were then analyzed by flow cytometry (Accuri C6; BD). The data were analyzed with Flowjo software.

Soft agar clonogenic assay. Five hundred cells were mixed with $0.3 \%$ Noble agar in growth medium and plated into 6 -well plates containing a solidified bottom layer $(0.6 \%$ Noble agar in growth medium). After 21 days of cell growth, colonies were stained with $5 \mathrm{mg} / \mathrm{ml}$ MTT (Sigma), photographed and recorded.

In vivo tumorigenic assay. $\mathrm{BE}(2)-\mathrm{C}$ cells were grown to $70-80 \%$ confluency and trypsinized. Cells $\left(1 \times 10^{6}\right.$ in $200 \mu$ l DMEM) were injected into the flanks of NOD/SCID mice. After one week of tumor growth, the mice were administered intraperitoneal injections of artemisinin at $100 \mathrm{mg} / \mathrm{kg}$ (mouse body weight) daily or vehicle control DMSO (1 $\mu \mathrm{l} / \mathrm{ml}$ DMEM) (16) for 12 days. Tumor diameter was measured with digital calipers every 3 days, and the tumor volume (V) was calculated by the formula: $\mathrm{V}=1 / 2$ (length $\mathrm{x}$ width ${ }^{2}$ ). After treatment, mice were sacrificed by $\mathrm{CO}_{2}$, and tumors were observed and weighed. All animal experiments were pre-approved by the Institutional Animal Care and Use Committee of Chongqing University.

Quantification and statistical analysis. Quantitative data are expressed as the mean \pm SD. The two-tailed Student's t-test was performed for paired samples. A minimum of three independent experiments was performed. Differences were considered statistically significant at $\mathrm{P}<0.05$.

\section{Results}

Artemisinin significantly inhibits cell proliferation in neuroblastoma cells. Neuroblastoma cells were treated with various concentrations of artemisinin, from $100 \mu \mathrm{M}$ to $400 \mu \mathrm{M}$, for an indicated period of time. As shown in Fig. 1A, a concentrationand time-dependent response to artemisinin in neuroblastoma 
A
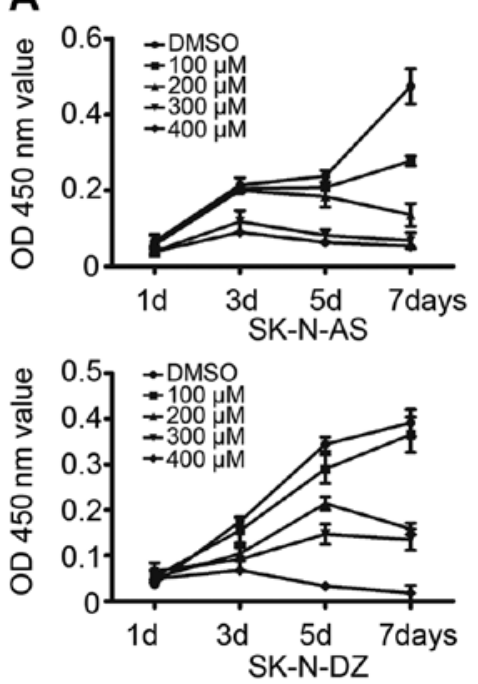
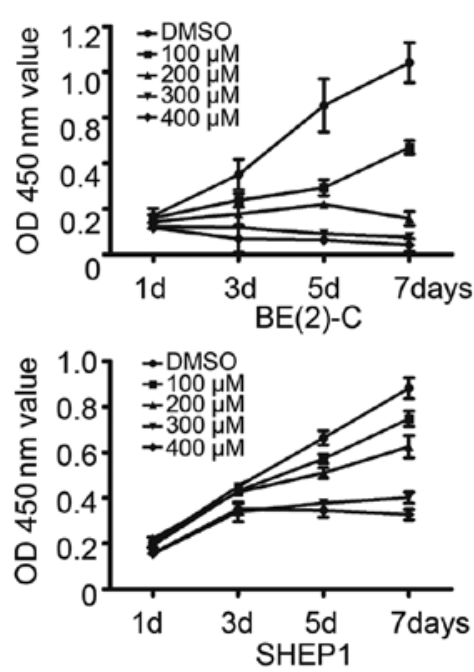

C

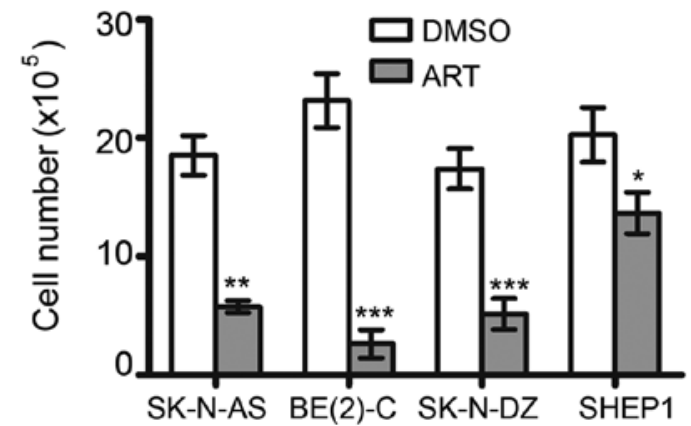

B
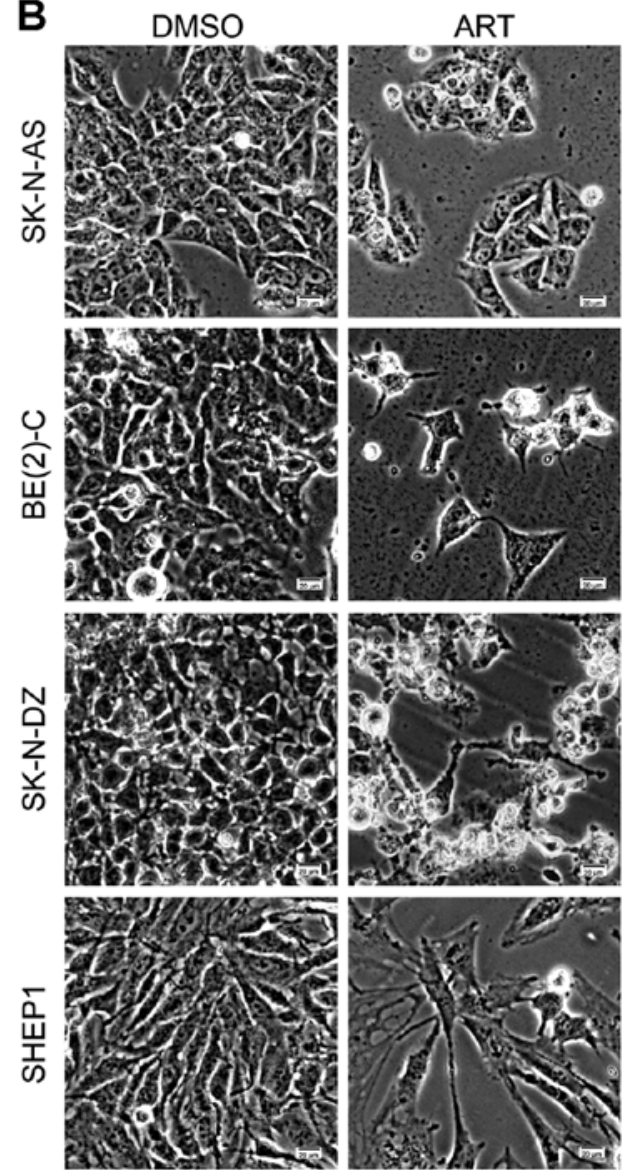

Figure 1. Artemisinin inhibits the cell growth of neuroblastoma cells. (A) SK-N-AS, BE(2)-C, SK-N-DZ and SHEP1 cells were treated with 100, 200, 300 and $400 \mu \mathrm{M}$ artemisinin; DMSO was used as a control. Cell growth was assessed by the CCK-8 assay every 2 days. (B) Morphologic examination of neuroblastoma cell growth after treated with $300 \mu \mathrm{M}$ artemisinin or DMSO for $72 \mathrm{~h}$ respectively. Scale bar, $20 \mu \mathrm{m}$. (C) The cell number of SK-N-AS, BE(2)-C, SK-N-DZ and SHEP1 cells was determined after artemisinin treatment at $300 \mu \mathrm{M}$ for $72 \mathrm{~h}$; DMSO was used as a control. In A and C, data represent the average \pm SD of at least three independent experiments. ${ }^{*} \mathrm{P}<0.05,{ }^{* *} \mathrm{P}<0.001,{ }^{* * *} \mathrm{P}<0.001$. ART, artemisinin.

cells was observed. Artemisinin markedly inhibited cell proliferation in the neuroblastoma cells. After $72 \mathrm{~h}$ of treatment with $300 \mu \mathrm{M}$ artemisinin, both morphologic examination and cell counting revealed that artemisinin significantly reduced cell growth in all four neuroblastoma cell lines (Fig. 1B and C). As a proliferation marker, Ki67 staining also confirmed that artemisinin markedly inhibited cell proliferation (Fig. 2A). The histogram statistics of the Ki67-positive rates also demonstrated that artemisinin was an effective agent for inhibiting the proliferation of neuroblastoma cells (Fig. 2B). The percentage of Ki67-positive SK-N-AS cells was reduced from 67.2 2.76 to $25.2 \pm 6.11 \%$. The percentage of Ki67-positive $\mathrm{BE}(2)-\mathrm{C}$ cells was reduced from $72.8 \pm 4.94$ to $22.7 \pm 1.51 \%$. The percentage of Ki67-positive SK-N-DZ cells was reduced from $82.1 \pm 4.89$ to $41.1 \pm 5.08 \%$, and the percentage of Ki67-positive SHEP1 cells was reduced from $57.6 \pm 1.88$ to $37.9 \pm 4.85 \%$.

Artemisinin treatment induces G1 phase cell cycle arrest. Furthermore, to gain insight into artemisinin-induced inhibition of cell proliferation, we examined the cell cycle distribution of the four neuroblastoma cell lines. After artemisinin treatment at $300 \mu \mathrm{M}$ for $72 \mathrm{~h}$, the proportion of cells in the $\mathrm{G} 1$ phase was significantly increased in all four cell lines (Fig. 3A). The cell cycle analysis of artemisinin-treated $\mathrm{BE}(2)-\mathrm{C}$ cells revealed a significant increase in the proportion of cells in the G0/G1 phase $(54.46 \pm 2.14 \%)$, compared with the vehicle-treated cells (G0/G1, 37.53 $\pm 1.82 \%$ ). Similar results were obtained in the SK-N-AS, SK-N-DZ and SHEP1 cell lines (Fig. 3B). These data demonstrated that artemisinin induced cell cycle arrest in the G1 phase. Western blot analysis also showed that artemisinin led to a marked downregulation of cyclinD1, CDK4 and cyclinE2 (Fig. 3C), which are collectively required for cell cycle progression through the G1 to $\mathrm{S}$ phases $(23,24)$. These data suggest that artemisinin inhibits cell proliferation through cell cycle arrest in neuroblastoma cells.

Artemisinin accelerates apoptosis in neuroblastoma cells. To investigate whether artemisinin induces apoptosis in neuroblastoma cells, SK-N-AS, BE(2)-C, SK-N-DZ, and SHEP1 cell lines were incubated with $300 \mu \mathrm{M}$ artemisinin for $72 \mathrm{~h}$. Trypan blue assay indicated that the cell death was increased after artemisinin incubation when compared with the control (Fig. 4A). Notably, treatment with artemisinin resulted in a higher proportion of cells with positive Annexin $\mathrm{V}$ and/or PI staining. Artemisinin markedly induced apoptosis in all four neuroblastoma cell lines (Fig. 4B), which indicated that artemisinin induced cell death through apoptosis in neuroblastoma cells. 
A

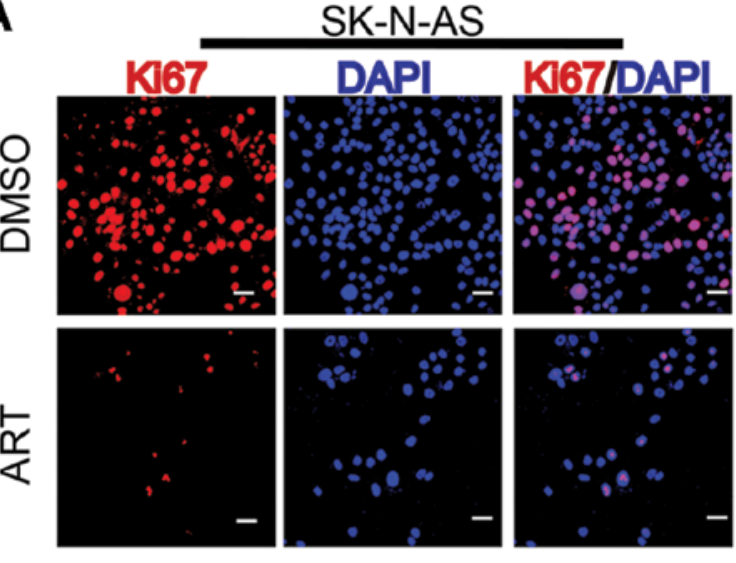

SK-N-AS

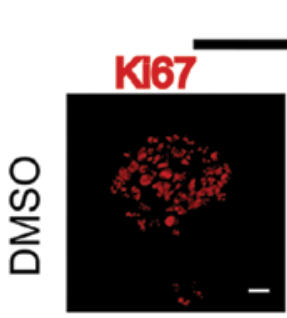

SK-N-DZ

DAPI
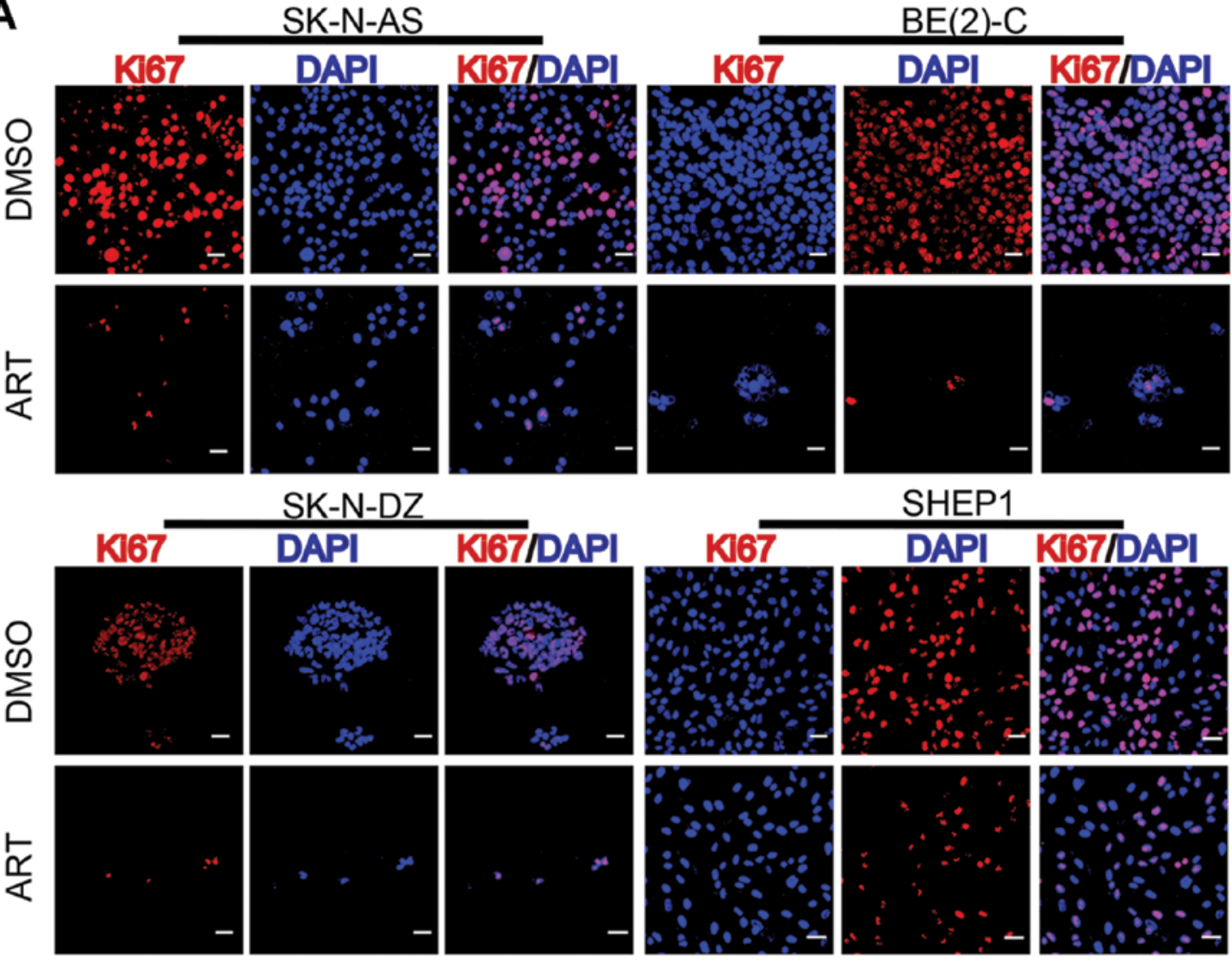

SHEP1
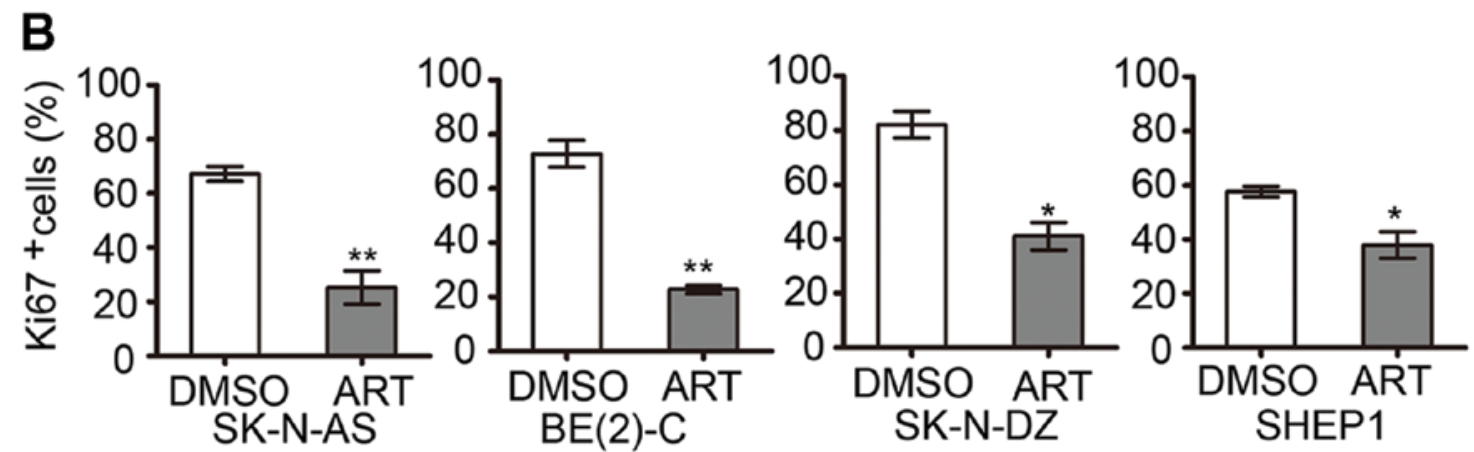

Figure 2. Artemisinin inhibits the cell proliferation of neuroblastoma cells. (A) SK-N-AS, BE(2)-C, SK-N-DZ and SHEP1 cells were grown on coverslips and treated with either DMSO or $300 \mu \mathrm{M}$ artemisinin for $72 \mathrm{~h}$, respectively. Cells were stained with an antibody against Ki67 (red), and counterstained with DAPI (blue); scale bar, $25 \mu \mathrm{m}$. (B) The percentage of $\mathrm{Ki}^{+} 7^{+}$cells was determined in a $\mathrm{x} 20$ field view under a microscope. Data represent the average \pm SD of at least three independent experiments. Statistical analysis was performed using the two-tailed Student's t-test. ${ }^{*} \mathrm{P}<0.05$, ${ }^{* *} \mathrm{P}<0.01$. ART, artemisinin.

Artemisinin suppresses the tumorigenicity in neuroblastoma cells. Soft agar is used to test the ability of single cancer cells to proliferate and form colonies, and is also used as a 'human tumor stem-cell assay' (25). In the present study, BE(2)-C cells treated with $300 \mu \mathrm{M}$ ART were observed to give rise to small and scant colonies in soft agar compared with the cells treated with DMSO (Fig. 5A and B). To determine the effect of artemisinin on tumorigenicity in neuroblastoma cells, we carried out a xenograft study in NOD/SCID mice. $\mathrm{BE}(2)-\mathrm{C}$ cells were implanted subcutaneously into the flanks of NOD/SCID mice. One week after tumor injection, mice were treated intraperitoneally with either DMSO or artemisinin at $100 \mathrm{mg} / \mathrm{kg}$ daily for 12 days. The volume and weight of the xenograft tumors in the artemisinin treatment group were much smaller and lighter than those in the DMSO group (Fig. 5C and D). These data indicate that artemisinin significantly suppresses the tumorigenicity of neuroblastoma cells.

\section{Discussion}

Neuroblastoma is a malignant pediatric tumor with a wide range of stages, requiring a wide range of therapeutic options. However, successful therapeutic options remain limited. Therefore, it is urgently necessary to identify additional chemotherapeutic agents to target this disease. It has been reported that artemisinin has multiple anti-proliferative activity, including cell growth suppression $(26,27)$, apoptosis induction (28), 

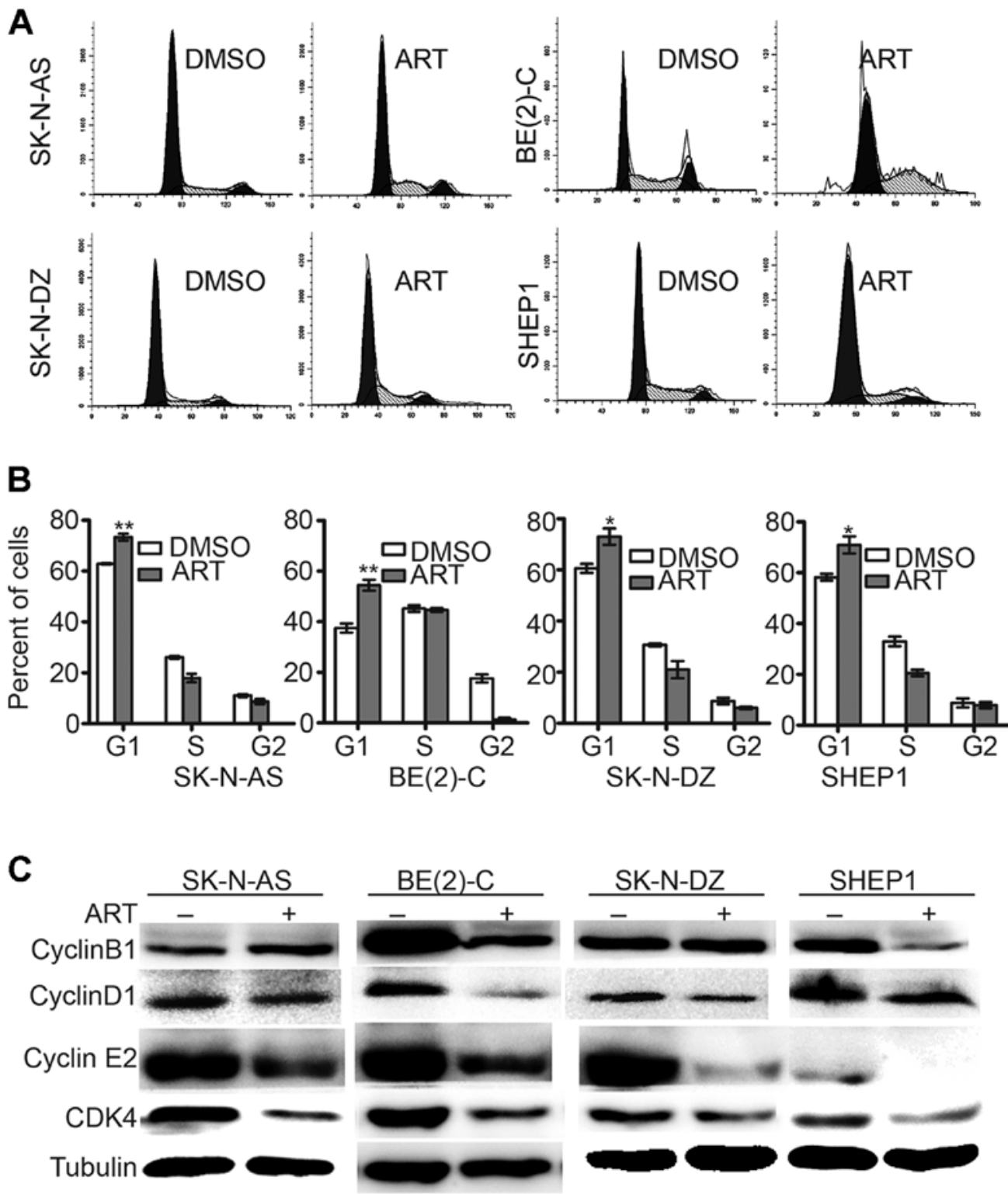

Figure 3. Artemisinin induces G1 phase cell cycle arrest in neuroblastoma cells. (A) SK-N-AS, BE(2)-C, SK-N-DZ and SHEP1 cells were treated with $300 \mu$ M artemisinin or DMSO for $72 \mathrm{~h}$. Cells were harvested, fixed with ethanol and stained with propidium iodide, DNA content was determined by flow cytometry. (B) The statistical analysis of the percentage of cells in each cell cycle phase in the four neuroblastoma cell lines is shown. Data represent the average \pm SD of at least three independent experiments. Statistical analysis was performed using the two-tailed Student's t-test. ${ }^{*} \mathrm{P}<0.05$, ${ }^{* * *} \mathrm{P}<0.01$. (C) Western blot analysis of cyclinB1, cyclinD1, cyclinE2 and CDK4 in neuroblastioma cells treated with artemisinin or DMSO for $72 \mathrm{~h}$. $\alpha$-Tubulin levels are shown as loading control. ART, artemisinin.

angiogenesis inhibition, cell migration disruption (29-31), and modulation of nuclear receptor responsiveness $(32,33)$. Yet, the effect of artemisinin on neuroblastoma remains unclear. The results presented in the present study demonstrated that artemisinin led to significantly decreased cell growth and cell proliferation, and increased apoptosis in neuroblastoma cells. We first demonstrated that artemisinin treatment suppressed the ability of colony formation in vitro and tumorigenicity of neuroblastoma cells in vivo.

Artemisinin has been suggested to promote cytostasis by G0/G1-phase arrest and to decrease the expression level of cyclinB1, CDK2 and CDC25A in colon cancer (34), and to inhibit the promoter activity of CDK4 in prostate cancer (16). Artemisinin also induced G2/M phase arrest in osteosarcoma cells (35). Our data indicated that artemisinin induced cell cycle arrest at the G1 phase, together with a decrease in the expression levels of cyclinD1, CDK4 and cyclinE2 in all four neuroblastoma cell lines. cyclinB1 was downregulated only in the BE(2)-C and SHEP1 cells, but no significant difference was noted in the SK-N-AS and SK-N-DZ cells.

Previous studies have shown that artemisinin induced apoptosis in pancreatic tumor cells (36), lung adenocarcinoma cells (37), liver cancer cells (38), and non-small cell lung cancer cells (39). In the present study, we first demonstrated that artemisinin induced the cell death and apoptosis of neuroblastoma cells at a lower dose than that for clinical usage. Next, we investigated the mechanism and the expression of apoptotic relevant proteins.

Collectively, our results revealed that artemisinin inhibited cell proliferation and tumor growth, with cell cycle arrest and 


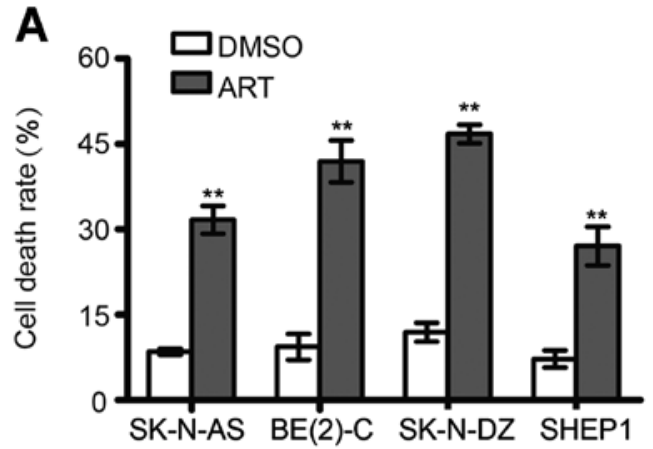

Figure 4. Artemisinin increases cell death and apoptosis in neuroblastoma cells. (A) SK-N-AS, BE(2)-C, SK-N-DZ and SHEP1 cells were treated with $300 \mu \mathrm{M}$ artemisinin or DMSO for $72 \mathrm{~h}$ and stained with trypan blue dye. The percentage of cell death was analyzed by the TC10 ${ }^{\mathrm{TM}}$ Automated Cell Counter. (B) Cells were treated with $300 \mu \mathrm{M}$ artemisinin or DMSO for $72 \mathrm{~h}$, and then cell apoptosis was determined using Annexin V FITC/propidium iodide staining. The statistical analysis of the percentage of apoptosis is shown. Data represent the average $\pm \mathrm{SD}$ of at least three independent experiments. Statistical analysis was performed using the two-tailed Student's t-test. ${ }^{* *} \mathrm{P}<0.01$. ART, artemisinin.
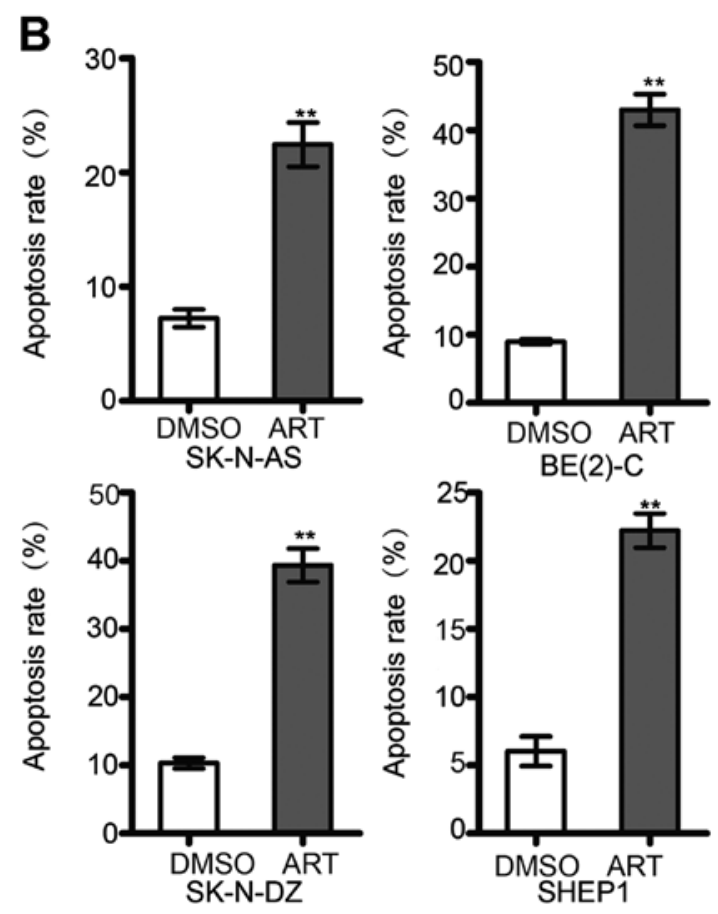

A

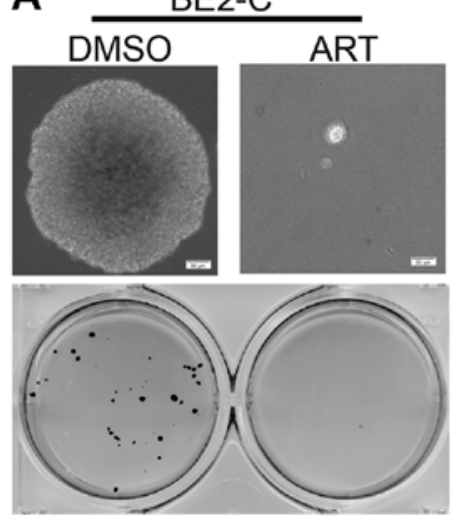

C

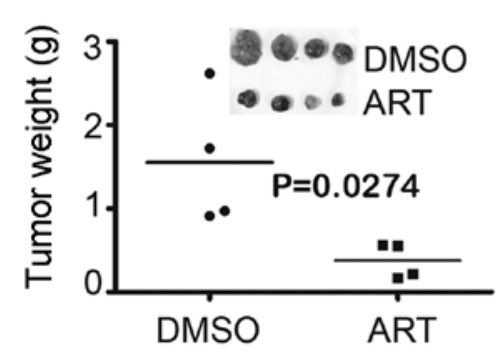

B

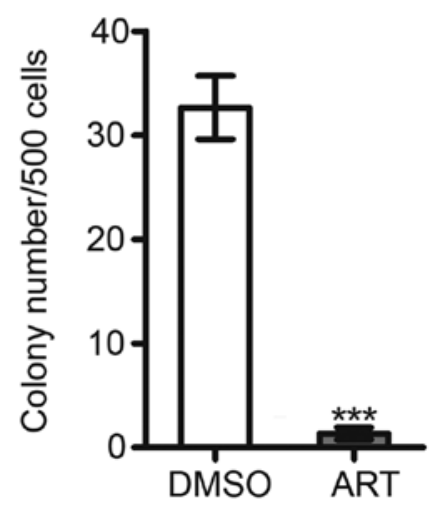

D

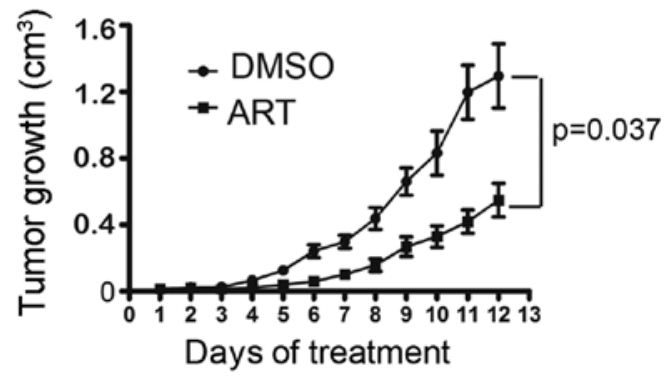

Figure 5. Artemisinin suppresses colony formation in vitro and tumor growth in vivo. (A) BE(2)-C cells were plated at 5x10² cells per well in 6-well culture plates and treated with $300 \mu \mathrm{M}$ artemisinin or DMSO in a soft agar culture system for 21 days. The colonies were then stained with MTT and imaged in order to determine the number of colonies. Scale bar, $50 \mu \mathrm{m}$. (B) Colonies $>0.5 \mathrm{~mm}$ or containing $>50$ cells were recorded and counted. Data represent the average \pm SD of at least three independent experiments. Statistical analysis was performed using the two-tailed Student's t-test. ${ }^{* *} \mathrm{P}<0.01$. (C) The NOD/SCID mouse xenograft tumors after treatment with DMSO or $100 \mathrm{mg} / \mathrm{kg}$ daily artemisinin were photographed and weighed. (D) Xenograft tumor volume was analyzed per group. In C and D, data were analyzed with two-tailed Student's t-test with the P-value as indicated. ART, artemisinin.

apoptosis induction in neuroblastoma cells. Since artemisinin has been used for the treatment of malaria for an extensive period of time, a large body of data regarding clinical tests and adverse drug reactions in patients are available. Therfore, 
artemisinin may serve as a potential new therapeutic agent for the treatment of neuroblastoma.

\section{Acknowledgements}

This research was supported by the National Basic Research Program of China (no. 2012cb114603), the National Natural Science Foundation of China (no. 31200223), the Research Fund for the Doctoral Program of Higher Education of China (no. 20130182110003) and the Natural Science Foundation of Chongqing (cstc2013jcyjys0007).

\section{References}

1. Brodeur GM: Neuroblastoma: biological insights into a clinical enigma. Nat Rev Cancer 3: 203-216, 2003.

2. Beckwith JB and Martin RF: Observations on the histopathology of neuroblastomas. J Pediatr Surg 3: 106-110, 1968.

3. Zhu S, Yan X, Xiang Z, Ding HF and Cui H: Leflunomide reduces proliferation and induces apoptosis in neuroblastoma cells in vitro and in vivo. PLoS One 8: e71555, 2013.

4. Li T, Cui ZB, Ke XX, et al: Essential role for p53 and caspase-9 in DNA damaging drug-induced apoptosis in neuroblastoma IMR32 cells. DNA Cell Biol 30: 1045-1050, 2011.

5. Li T, Wang L, Ke XX, et al: DNA-damaging drug-induced apoptosis sensitized by $\mathrm{N}-\mathrm{myc}$ in neuroblastoma cells. Cell Biol Int 36: 331-337, 2012.

6. Askin FB and Perlman EJ: Neuroblastoma and peripheral neuroectodermal tumors. Am J Clin Pathol 109: S23-S30, 1998.

7. Shah S and Ravindranath Y: Neuroblastoma. Indian J Pediat 65 : 691-705, 1998.

8. Bessho F: Incidence of neuroblastoma. Lancet 353: 70, 1999.

9. Sridhar S, Al-Moallem B, Kamal H, Terrile M and Stallings RL: New insights into the genetics of neuroblastoma. Mol Diagn Ther 17: 63-69, 2013.

10. Crespo-Ortiz MP and Wei MQ: Antitumor activity of artemisinin and its derivatives: from a well-known antimalarial agent to a potential anti-cancer drug. J Biomed Biotechnol 2012: 247597 2012.

11. Newman DJ and Cragg GM: Natural products as sources of new drugs over the last 25 years. J Nat Prod 70: 461-477, 2007.

12. Zhang YJ, Gallis B, Taya M, Wang S, Ho RJ and Sasaki T: $\mathrm{pH}$-responsive artemisinin derivatives and lipid nanoparticle formulations inhibit growth of breast cancer cells in vitro and induce down-regulation of HER family members. PloS One 8 e59086, 2013.

13. Miller LH and Su X: Artemisinin: discovery from the Chinese herbal garden. Cell 146: 855-858, 2011.

14. Sundar SN, Marconett CN, Doan VB, Willoughby JA Sr and Firestone GL: Artemisinin selectively decreases functional levels of estrogen receptor-alpha and ablates estrogen-induced proliferation in human breast cancer cells. Carcinogenesis 29: 2252-2258, 2008.

15. Duffy PE and Mutabingwa TK: Drug combinations for malaria: time to ACT? Lancet 363: 3-4, 2004.

16. Willoughby JA Sr, Sundar SN, Cheung M, Tin AS, Modiano J and Firestone GL: Artemisinin blocks prostate cancer growth and cell cycle progression by disrupting Sp1 interactions with the cyclin-dependent kinase-4 (CDK4) promoter and inhibiting CDK4 gene expression. J Biol Chem 284: 2203-2213, 2009.

17. Lai HC, Singh NP and Sasaki T: Development of artemisinin compounds for cancer treatment. Invest New Drugs 31: 230-246, 2013.

18. Tin AS, Sundar SN, Tran KQ, Park AH, Poindexter KM and Firestone GL: Antiproliferative effects of artemisinin on human breast cancer cells requires the downregulated expression of the E2F1 transcription factor and loss of E2F1-target cell cycle genes. Anticancer Drugs 23: 370-379, 2012.
19. Weifeng T, Feng S, Xiangji L, et al: Artemisinin inhibits in vitro and in vivo invasion and metastasis of human hepatocellular carcinoma cells. Phytomedicine 18: 158-162, 2011.

20. Reungpatthanaphong $P$ and Mankhetkorn S: Modulation of multidrug resistance by artemisinin, artesunate and dihydroartemisinin in K562/adr and GLC4/adr resistant cell lines. Biol Pharm Bull 25: 1555-1561, 2002.

21. Yamachika E, Habte T and Oda D: Artemisinin: an alternative treatment for oral squamous cell carcinoma. Anticancer Res 24: 2153-2160, 2004.

22. Cui H, Schroering A and Ding HF: p53 mediates DNA damaging drug-induced apoptosis through a caspase-9-dependent pathway in SH-SY5Y neuroblastoma cells. Mol Cancer Ther 1: 679-686, 2002.

23. Lim S and Kaldis P: Cdks, cyclins and CKIs: roles beyond cell cycle regulation. Development 140: 3079-3093, 2013.

24. Lee IH and Finkel T: Metabolic regulation of the cell cycle. Curr Opin Cell Biol 25: 724-729, 2013.

25. Agrez M, Kovach J and Lieber M: Cell aggregates in the soft agar 'human tumour stem-cell assay'. Br J Cancer 46: 880, 1982.

26. Youns M, Efferth T, Reichling J, Fellenberg K, Bauer A and Hoheisel JD: Gene expression profiling identifies novel key players involved in the cytotoxic effect of Artesunate on pancreatic cancer cells. Biochem Pharmacol 78: 273-283, 2009.

27. Li S, Xue F, Cheng Z, et al: Effect of artesunate on inhibiting proliferation and inducing apoptosis of SP2/0 myeloma cells through affecting NFkappaB p65. Int J Hematol 90: 513-521, 2009.

28. Zeng Y, Ni X, Meng WT, Wen Q and Jia YQ: Inhibitive effect of artesunate on human lymphoblastic leukemia/lymphoma cells. Sichuan Da Xue Xue Bao Yi Xue Ban 40: 1038-1043, 2009 (In Chinese).

29. Zhou HJ, Wang WQ, Wu GD, Lee J and Li A: Artesunate inhibits angiogenesis and downregulates vascular endothelial growth factor expression in chronic myeloid leukemia K562 cells. Vascul Pharmacol 47: 131-138, 2007.

30. Li LN, Zhang HD, Yuan SJ, Yang DX, Wang L and Sun ZX: Differential sensitivity of colorectal cancer cell lines to artesunate is associated with expression of beta-catenin and E-cadherin. Eur J Pharmacol 588: 1-8, 2008.

31. Rasheed SAK, Efferth T, Asangani IA and Allgayer H: First evidence that the antimalarial drug artesunate inhibits invasion and in vivo metastasis in lung cancer by targeting essential extracellular proteases. Int J Cancer 127: 1475-1485, 2010.

32. Chaturvedi D, Goswami A, Saikia PP, Barua NC and Rao PG: Artemisinin and its derivatives: a novel class of anti-malarial and anticancer agents. Chem Soc Rev 39: 435-454, 2010.

33. Firestone GL and Sundar SN: Anticancer activities of artemisinin and its bioactive derivatives. Expert Rev Mol Med 11: e32, 2009.

34. Efferth T, Sauerbrey A, Olbrich A, et al: Molecular modes of action of artesunate in tumor cell lines. Mol Pharmacol 64: 382-394, 2003.

35. Xu Q, Li ZX, Peng HQ, et al: Artesunate inhibits growth and induces apoptosis in human osteosarcoma HOS cell line in vitro and in vivo. J Zhejiang Univ Sci B 12: 247-255, 2011.

36. Noori S, Hassan ZM and Farsam V: Artemisinin as a Chinese medicine, selectively induces apoptosis in pancreatic tumor cell line. Chin J Integr Med: Jun 15, 2013 (Epub ahead of print).

37. Xiao F, Gao W, Wang $X$ and Chen T: Amplification activation loop between caspase- 8 and -9 dominates artemisinin-induced apoptosis of ASTC-a-1 cells. Apoptosis 17: 600-611, 2012.

38. Hou JM, Wang DS, Zhang RW and Wang H: Experimental therapy of hepatoma with artemisinin and its derivatives: In vitro and in vivo activity, chemosensitization, and mechanisms of action. Clin Cancer Res 14: 5519-5530, 2008.

39. Gao W, Xiao F, Wang X and Chen T: Artemisinin induces A549 cell apoptosis dominantly via a reactive oxygen species-mediated amplification activation loop among caspase-9, -8 and -3 . Apoptosis 18: 1201-1213, 2013. 